\title{
Bioconductor caAffy Invariant Set Normalization
}

National Cancer Institute

\section{Source}

National Cancer Institute. Bioconductor caAffy Invariant Set Normalization. NCI

Thesaurus. Code C63932.

A method to normalize arrays in an AffyBatch using an invariant set, as defined in documents available at http://bioconductor.org/packages/1.9/bioc/html/affy.html 